\title{
Proof of concept for a wrist mechanism for articulated forceps for use in robot-assisted laparoscopic surgery
}

\author{
Makoto Jinno* (1)
}

\begin{abstract}
Laparoscopic surgery is a minimally invasive surgery that accelerates postoperative recovery, but it can only be performed by surgeons with advanced surgical skills. One of the main difficulties in laparoscopic surgery is the restriction of free motion of the forceps because of the limited degrees of freedom by the trocar. Recently, many master-slave manipulators with articulated forceps have been used in laparoscopic surgery to solve this problem. The wrist mechanism of an articulated forceps affects the controllability and range of motion of a slave manipulator in a patient's abdominal cavity. Therefore, it is important to improve upon the wrist mechanism of articulated forceps for robot-assisted laparoscopic surgery. This paper aims to propose a new wrist mechanism for articulated forceps to be used in laparoscopic surgery. The degrees of freedom of the proposed design are three motor-driven axes that use wires and pulleys (a pitch axis, yaw axis, and gripper axis) and a roll axis driven by manual rotation of the forceps shaft. In the proposed mechanism, the offset distance between the pitch and yaw axes was reduced. The offset value, i.e., $5.95 \mathrm{~mm}$, is shorter than the maximum outer diameter $7.5 \mathrm{~mm}$ of the wrist mechanism. Singularity configurations of the wrist axes are avoided near the standard working posture by configuring the pitch and yaw axis. There are few parts and it is simple as compared to the previously developed clinical use robotic forceps. Furthermore, the effectiveness of the proposed mechanism was verified by tests. The range of pitch motion is $\pm 90^{\circ}$, yaw motion is $\pm 85^{\circ}$ (or $\pm 90^{\circ}$ ), and gripper motion is $60^{\circ}$ (or $50^{\circ}$ ). The transmission efficiencies are from about 55 to $75 \%$. The grasping force and torque are more than $5 \mathrm{~N}$ and $70 \mathrm{mNm}$.
\end{abstract}

Keywords: Surgical robotics, Minimally invasive surgery, Wire drive mechanism, Forceps, Master-slave manipulator

\section{Background}

Laparoscopic surgery is one of the most commonly used minimally invasive surgical techniques. As shown in Fig. 1's depiction of conventional laparoscopic surgery, a surgeon operates using forceps passed through trocars (each approximately $5-10 \mathrm{~mm}$ in diameter) into a patient's abdominal cavity while watching an image acquired by a laparoscope. Since only a small incision is made, the patient can recover sooner and overall medical costs are reduced. Therefore, laparoscopic surgery has great advantages for the patient and has become widespread in recent years [1]. However, laparoscopic surgery

*Correspondence: mjinno@kokushikan.ac.jp

School of Science and Engineering, Kokushikan University, 4-28-1, Setagaya, Setagaya-ku, Tokyo 154-8515, Japan can only be performed by surgeons with advanced skills. One of the main difficulties in laparoscopic surgery is the restriction of free motion of the forceps because of the size of the trocar. The position and posture of the gripper of the forceps cannot be changed freely in the patient's abdominal cavity during the procedure because the shaft of the forceps is restricted by the trocar. Recently, many master-slave manipulators with articulated forceps have been used in laparoscopic surgery to solve this problem [2]. Compact and low-cost manual or motor-driven articulated forceps have also been developed for clinical use [3-6].

The wrist mechanism of an articulated forceps affects the controllability and range of motion of a slave manipulator in a patient's abdominal cavity. Therefore, it is 


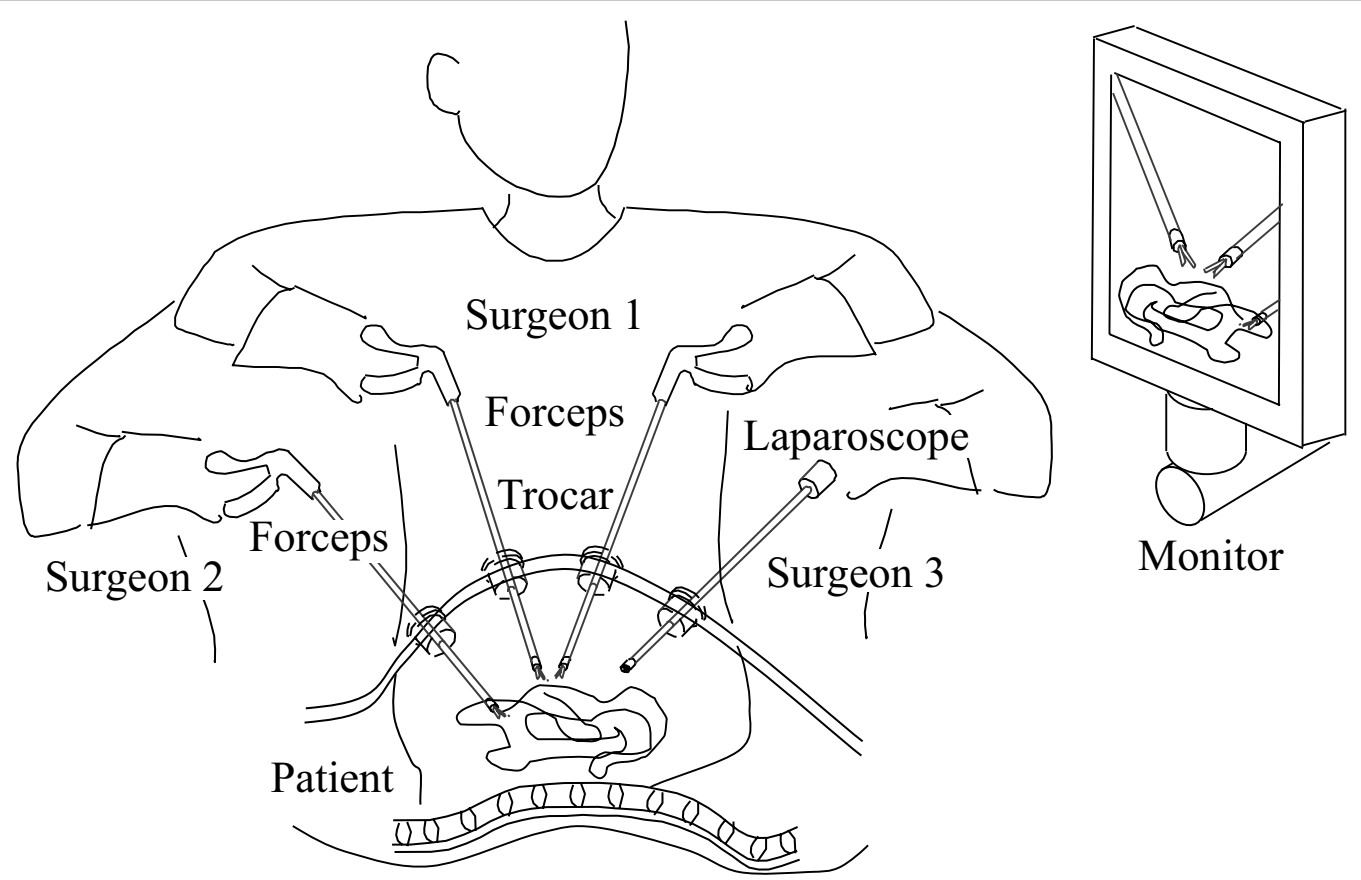

Fig. 1 Conventional laparoscopic surgery

important to improve upon the wrist mechanism of articulated forceps for robot-assisted laparoscopic surgery. Several wrist mechanisms for articulated forceps have been proposed.

The wrist mechanism proposed by da Vinci [2] adopts a wire-pulley drive mechanism [7]. Thielmann et al. and Tadano et al. have also proposed a wire-pulley drive mechanism $[8,9]$. In the wire-pulley drive mechanism, the mechanical design about the arrangement of pulleys and routing of wires is very important to keep the curvature radius of wires and to reduce distance between joints.

The wrist mechanism proposed by Kymerax [6] adopts a combination of wire-pulley drive and gear drive mechanism [10]. Further, we have proposed another type of wire-pulley and gear drive mechanism [11, 12]. Nishizawa and Kobayashi proposed a non-interference mechanism using wire-pulley and gears mechanism [13]. In this mechanism, in addition to wire-pulley design, strength design of gears and manufacturing accuracy of parts to reduce gear backlash are very important.

The wrist mechanism of the Radius [5] adopts the combination of torque tube, gear, and link drive mechanism [14]. Also, Fujii et al. [15] proposed a torque tube and gear drive mechanism. Similarly, strength design of gears and manufacturing accuracy of parts to reduce gear backlash in torque tube and gear drive mechanism are very important.
The Autonomy [3] and the FlexDex [4] adopted a flexible bending joint and wire drive mechanism $[16,17]$. Haraguchi et al. [18] also proposed a flexible bending joint and wire drive mechanism using a spring for the joint. The bending mechanisms using flexible joint are very simple. Since the bending radius of the flexible joint is large with respect to the forceps diameter, large motion is required to determine the posture of the end effector. With a forceps using a flexible bending joint, the procedure in a narrow space is difficult.

Moreover, link drive mechanisms [19, 20] and parallel link mechanisms [21-23] are proposed. Ishii and Kobayashi propose a parallel link mechanism using doublescrew-drive mechanism [24]. Arata et al. [25] proposed a parallel link mechanism using spring links. It is difficult to obtain wide motion range of about $\pm 90^{\circ}$ for each joint by the link drive mechanisms. Also, link drive mechanisms need a lot of components and manufacturing accuracy of parts to reduce link backlash, and the cost increases.

Considering the need for a compact and maneuverable wrist mechanism for robot-assisted laparoscopic forceps, this study aims to propose a new wrist mechanism using wire-pulley drive mechanism. This paper first examines the most important specifications required for such a wrist mechanism. Next, a new wrist mechanism is proposed to satisfy those specifications, with a basic design and forward and inverse kinematics analyses. Third, a 
prototype model of the wrist mechanism is detailed. Finally, the paper reports the experimental results using a test bench to confirm the effectiveness of the mechanism.

\section{Methods}

This section presents the required specifications and the mechanical design of a wrist mechanism for articulated forceps used in robot-assisted laparoscopic surgery.

\section{Configuration of the degrees of freedom}

Generally, three axes are required to allow any posture of an end effector. In case of master-slave manipulators and articulated forceps for laparoscopic surgery, mostly, the first axis that is the most proximal side axis for the posture of the wrist axes is the roll axis of rotation of the forceps shaft by manual or motor drive to simplify the wrist mechanism. Generally, for the second and third axis for the posture of the wrist axes, combination of bending axis, roll axis, and curvature axis, among others are applied. There are three kinds of combinations of the second and third axis for clinical use articulated forcipes of laparoscopic surgery $[7,10,15-17]$. Also, the prototype models of wrist mechanisms for articulated forceps $[8,9$, $11-14,18-25]$ can be classified by the same three configurations. The three configurations of the second and third axes are as follows and showed in Fig. 2:

A. A combination of two bending axes (a pitch axis orthogonal to the shaft axis and a yaw axis, or vice versa) $[7,8,9,12,13,19,20]$.

B. A bending axis (pitch axis or yaw) and roll axis [10, $11,14,15]$.

C. Two curvature axes for up/down and right/left motions using joint ring, linkage, and spring, among others. This category includes parallel mechanism [16-18, 21-25].

Among the basic required specifications for the wrist mechanism, the following two items are especially important for master-slave manipulators for laparoscopic surgery.
1. A minimal offset distance between the pitch axis and yaw axis.

If the posture axis offset is large, when changing the posture of the end effector in a fixed state of a tool center point, the motion of the three basic axes for the positioning of the end effector increases. Therefore, the effective working area of the robot including the posture of the end effector cannot be secured effectively. In particular, in the case of a laparoscopic assist robot, since the three basic axes must be operated under the trocar restriction, large motion of a forceps shaft is needed to change the posture of the end effector in a fixed state of a tool center point. Therefore, the influence of the offset is more serious, and to secure a sufficient working area, the three basic axes need to be enlarged. Also, the offset would render the wrist mechanism too large for laparoscopic applications.

2. Avoidance of singularity of the wrist axes in the standard working posture.

It is necessary that the singularity posture is not close to the standard working posture. In case of laparoscopic surgery, it is natural to perform the procedure with the gripper and the forceps axes in near parallel, and surgeons use up/down or right/left motion of the gripper frequently. Therefore, it is desirable that the singularity of the wrist axes is sufficiently different from this configuration that is the gripper and the forceps axes in near parallel.

Table 1 list the comparison between these two design constraints with the three configurations of wrist axes described above.

A. The offset is difficult to eliminate by simple mechanism but can be reduced. The singularity configuration is not near the standard working posture [7-9, $12,13,19,20]$.

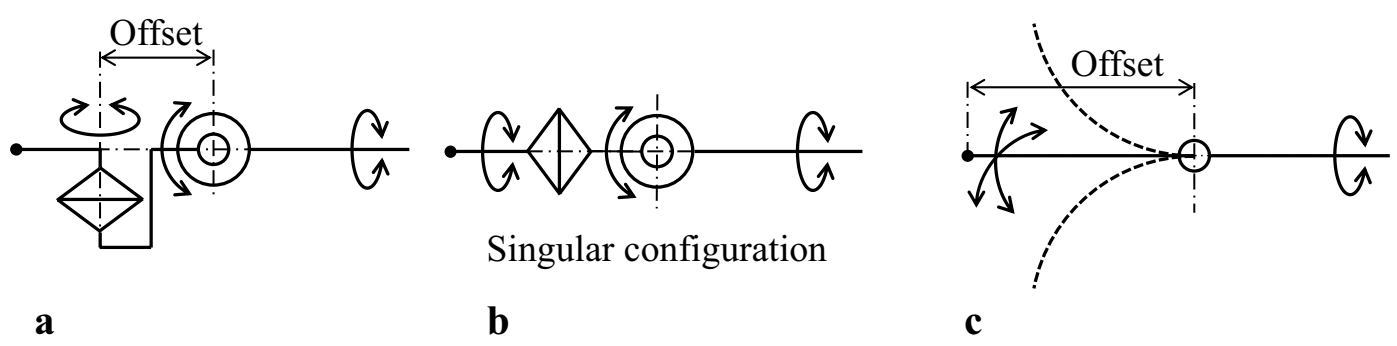

Fig. 2 Comparison of axis configurations: a pitch and yaw axes; $\mathbf{b}$ yaw and roll axes; $\mathbf{c}$ two bending axes 
Table 1 Comparison of axis configurations about offset between axes and the distance of standard working posture from singular configuration

\begin{tabular}{llll}
\hline Items & $\begin{array}{l}\text { A: } \\
\text { Pitch and yaw } \\
\text { axes }\end{array}$ & $\begin{array}{l}\text { B: } \\
\text { Yaw and roll } \\
\text { axes }\end{array}$ & $\begin{array}{l}\text { C: } \\
\mathbf{2} \text { bending axes }\end{array}$ \\
\hline $\begin{array}{l}\text { Offset between } \\
\text { axes }\end{array}$ & $\triangle$ & 0 & $\times$ \\
$\begin{array}{c}\text { Distance from } \\
\text { singular con- } \\
\text { figuration }\end{array}$ & $\bigcirc$ & $\times$ & 0 \\
\hline
\end{tabular}

It is possible to eliminate the offset, for example, using universal joint or ball joint. But the joint mechanisms are complex and the motion range is small.

B. Since the longitudinal directions of the gripper and forceps axes coincide with the roll axis, it is difficult to avoid the singularity configuration near the standard working posture. The offset is eliminated $[10,11$, $14,15]$

C. Since the posture is determined by bending motions, the offset of both bending mechanisms tends to be very large. The singularity configuration is not even close to the standard working posture [16-18, 2125].

Therefore, we designed a wrist mechanism that reduces the offset by simple mechanism with respect to the pitch and yaw axes.

\section{Mechanical design}

Figure 3 shows a conceptual design of the proposed wrist mechanism with pitch and yaw axes and a gripper. The pitch mechanism is a simple structure that transmits power from the drive pulley in the base (not shown the figure) to the driven pulley via a wire. Similarly, the yaw

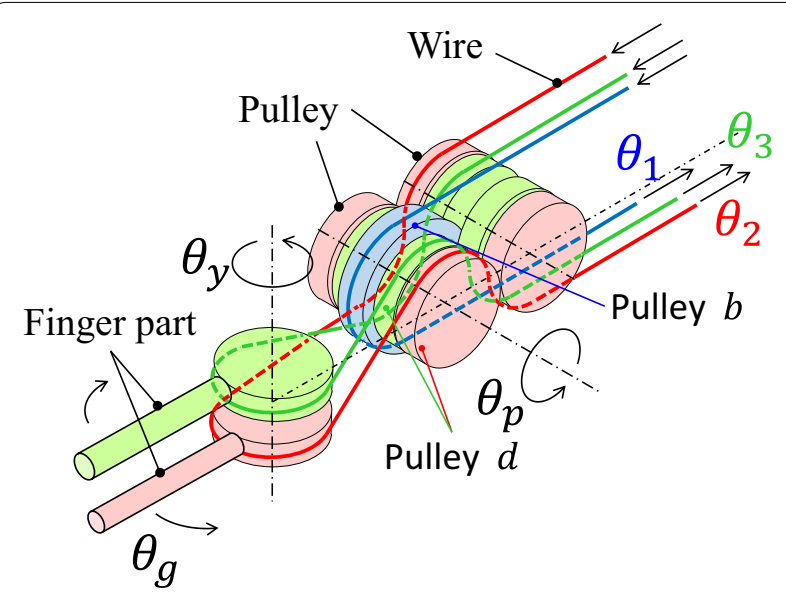

Fig. 3 Conceptual diagram of the wrist mechanism and gripper mechanisms are configured to transmit power from the drive pulleys on the base (not shown the figure) to the driven pulleys via wires, with idle pulleys to direct the wires through the pitch axis. Therefore, the motions of the yaw and gripper axis are performed by cooperative motion. Also, since the wires are flexible, the entry directions of the wires to pulleys and the rotation axis of the pulleys do not need to be orthogonal to each other. Figure 4 shows the basic design of the proposed wrist mechanism based on the conceptual design. Table 2 lists the specifications of the wrist mechanism and Table 3 lists its component parts. The maximum outer diameter is $7.5 \mathrm{~mm}$, which is equivalent to the conventional wrist mechanisms used in articulated forceps for robot-assisted laparoscopic surgery $[2,5]$. The offset distance between the pitch axis and the yaw axis is $5.95 \mathrm{~mm}$ and it is shorter than the maximum outer diameter $(7.5 \mathrm{~mm})$ of the wrist mechanism. Since mechanical parts such as idle pulleys and linkages are not needed between the pitch axis and the yaw axis, the offset distance can be reduced. Also, since all the three supporting shafts supporting the pulley, pitch part, and finger parts are supported stably at both ends of the shaft and the parts are in line with each other (coaxial), stresses on the support shafts are reduced, the pulleys and structural members are supported rigidly, and relatively few parts are needed. In addition, since the wires pass through the pitch axis on pulleys, bending stress on the wires and friction between the wires and pulleys are reduced. These qualities show that the proposed mechanism could function as a highly durable and efficient wrist mechanism for articulated forceps in robot-assisted laparoscopic surgery.

\section{Kinematics of the wrist mechanism}

Figure 5 shows the coordinate system for the drive mechanism of the proposed wrist mechanism. It is an interference-drive joint mechanism. The forward and inverse kinematics matrices are as follows:

$$
\begin{aligned}
& {\left[\begin{array}{l}
\theta_{p} \\
\theta_{y} \\
\theta_{g}
\end{array}\right]=\left[\begin{array}{ccc}
\frac{a}{b} & 0 & 0 \\
\frac{a}{b} \frac{d}{e} & \frac{c}{2 e} & \frac{c}{2 e} \\
0 & \frac{c}{e} & -\frac{c}{e}
\end{array}\right]\left[\begin{array}{l}
\theta_{1} \\
\theta_{2} \\
\theta_{3}
\end{array}\right]} \\
& {\left[\begin{array}{l}
\theta_{1} \\
\theta_{2} \\
\theta_{3}
\end{array}\right]=\left[\begin{array}{ccc}
\frac{b}{a} & 0 & 0 \\
-\frac{d}{c} & \frac{e}{c} & \frac{e}{2 c} \\
-\frac{d}{c} & \frac{e}{c} & -\frac{e}{2 c}
\end{array}\right]\left[\begin{array}{l}
\theta_{p} \\
\theta_{y} \\
\theta_{g}
\end{array}\right]}
\end{aligned}
$$

where $\mathrm{a}$ and $\mathrm{b}$ are the pitch diameters of the wire at the drive pulley and driven pulley of the pitch axis (first axis), respectively, and c, d, and e are the pitch diameters of the wires at drive pulleys, idle pulleys, and driven pulleys of 

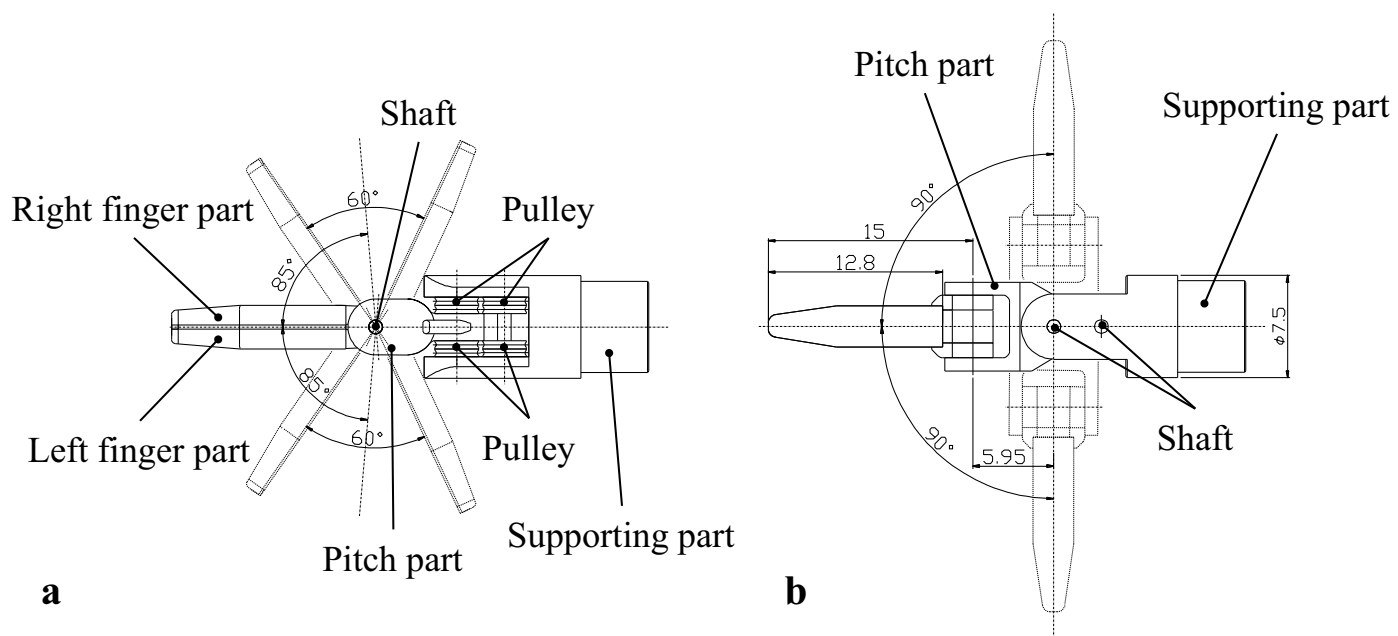

Fig. 4 Design of the wrist mechanism: a yaw and gripper axis range of motion (top view); $\mathbf{b}$ pitch axis range of motion (front view)

Table 2 Specifications of the wrist mechanism

\begin{tabular}{ll}
\hline Items & Specification \\
\hline Size & \\
Maximum diameter & $\varphi 7.5 \mathrm{~mm}$ \\
Offset from pitch axis to yaw axis & $5.95 \mathrm{~mm}$ \\
Offset from yaw axis to gripper axis & $0 \mathrm{~mm}$ \\
Radius from gripper axis to tip & $15 \mathrm{~mm}$ \\
Gripper face length & $12.8 \mathrm{~mm}$ \\
Range of motion & \\
Pitch & $\pm 90^{\circ}$ \\
Yaw & $\pm 85^{\circ}\left( \pm 90^{\circ}\right)$ \\
Gripper & $60^{\circ}\left(50^{\circ}\right)$ \\
\hline
\end{tabular}

Table 3 Component parts list of the wrist mechanism

\begin{tabular}{lc}
\hline Part name & Quantity \\
\hline Complex shape parts & \\
Supporting part & 1 \\
Pitch part & 1 \\
Right finger part & 1 \\
Left finger part & 1 \\
Simple shape parts & \\
Supporting shaft & 3 \\
Pulley $(\varphi 3(\mathrm{~mm}))$ & 8 \\
Stainless wire $(\varphi 0.45(\mathrm{~mm}), 7 \times 19)$ & 3 \\
Total & 18 \\
\hline
\end{tabular}

the yaw and gripper axes (second and third axes). The pitch diameters are the sum of the pulley diameter and wire diameter. Table 4 lists the pulley diameters and the pitch diameters. The diameters of the drive pulley are chosen with regard to the range of motion $\left( \pm 90^{\circ}\right)$ of the drive motor discussed in the next section. The suffixes $p$, $y$, and $g$ indicate yaw, pitch, and gripper axis. From the above equation, the relation between the driving torque and joint torque can be derived using the principle of virtual work:

$$
\begin{aligned}
& {\left[\begin{array}{l}
\tau_{1} \\
\tau_{2} \\
\tau_{3}
\end{array}\right]=\left[\begin{array}{ccc}
\frac{a}{b} & \frac{a}{b} \frac{d}{e} & 0 \\
0 & \frac{c}{2 e} & \frac{c}{e} \\
0 & \frac{c}{2 e} & -\frac{c}{e}
\end{array}\right]\left[\begin{array}{l}
\tau_{p} \\
\tau_{y} \\
\tau_{g}
\end{array}\right]} \\
& {\left[\begin{array}{l}
\tau_{p} \\
\tau_{y} \\
\tau_{g}
\end{array}\right]=\left[\begin{array}{ccc}
\frac{b}{a} & -\frac{d}{c} & -\frac{d}{c} \\
0 & \frac{e}{c} & \frac{e}{c} \\
0 & \frac{e}{2 c} & -\frac{e}{2 c}
\end{array}\right]\left[\begin{array}{l}
\tau_{1} \\
\tau_{2} \\
\tau_{3}
\end{array}\right]}
\end{aligned}
$$

Although the gripping and releasing torques in the gripper axis have no influence on the first axis driving torque, the yaw axis torque does interfere with the first axis driving torque. Therefore, the first axis drive system must be designed with enough drive torque and stiffness of the joints to overcome increases in angular moment when the yaw axis interferes with the pitch axis. To increase the joint stiffness, stiffer wires and large-diameter pulleys can be used. The kinematics of the proposed wrist mechanism is almost the same as conventional clinical use articulated forceps [7].

\section{Results and discussion} Prototype model

Based on the abovementioned mechanical design considerations, a prototype model of the wrist mechanism was developed. Manufacturing and assembly of the parts presented no problems. Figure 6 shows an overview of the wrist mechanism. 


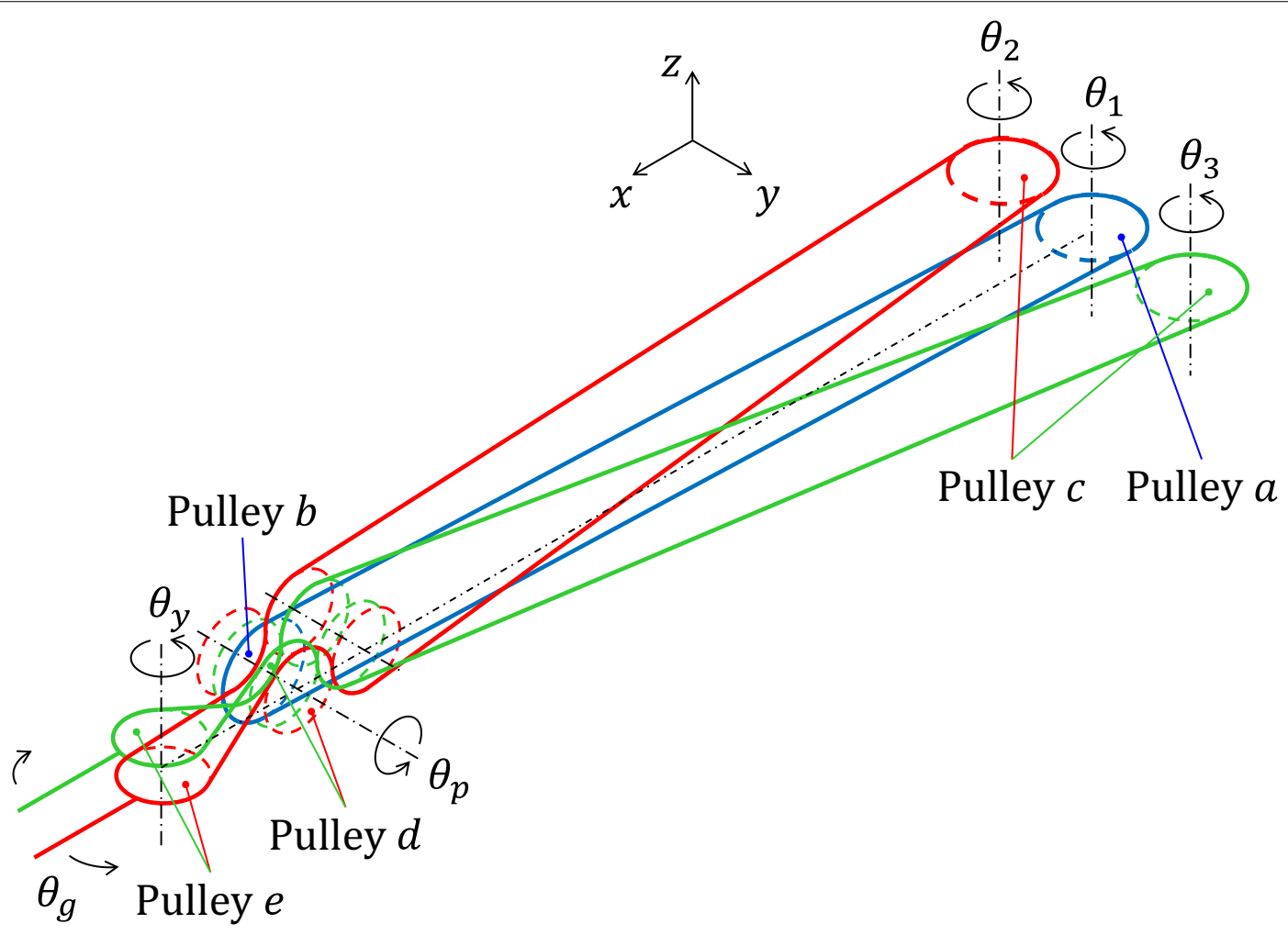

Fig. 5 Coordinate system

Table 4 Pulley diameter and pitch diameter

\begin{tabular}{lll}
\hline Pulley name & Pulley diameter $(\mathbf{m m})$ & Pitch diameter $(\mathbf{m m})$ \\
\hline$A$ & $\varphi 7$ & $\varphi 7.45$ \\
$B$ & $\varphi 4$ & $\varphi 4.45$ \\
$C$ & $\varphi 7$ & $\varphi 7.45$ \\
$D$ & $\varphi 3$ & $\varphi 3.45$ \\
$E$ & $\varphi 3$ & $\varphi 3.45$ \\
\hline
\end{tabular}

\section{Evaluation experiments}

\section{Results of range of motion}

Figure 7 shows the range of motion of the pitch, yaw, and gripper axis. These data show that the specifications listed in Table 2 have been satisfied.

\section{Results of transmission mechanism efficiency}

In the test shown in Fig. 8, the input torque $\left(R_{i} r_{i}\right)$ applied to the drive shaft pulley via the force gage and the output torque $\left(F_{o} r_{o}\right)$ were both measured by a force sensor. The force-transmission efficiencies $\eta$ for each axis were then calculated with the following equation:

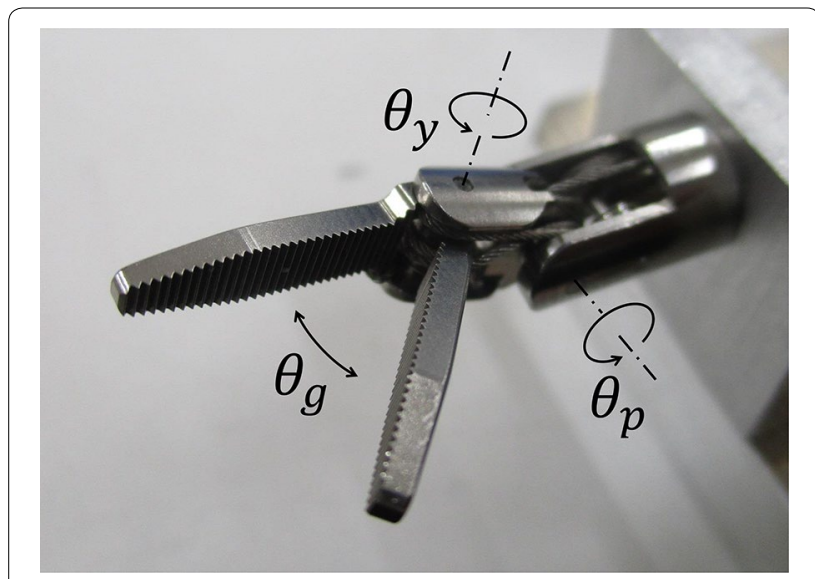

Fig. 6 Overview of the wrist mechanism of articulated forceps

$$
\eta=\frac{F_{o} r_{o}}{R F_{i} r_{i}}
$$

where $R$ is the reduction ratio (pulley ratio) of each axis. The input torque was about $200 \mathrm{mNm}$. While measuring the second and third axes, the pitch axis was fixed. 


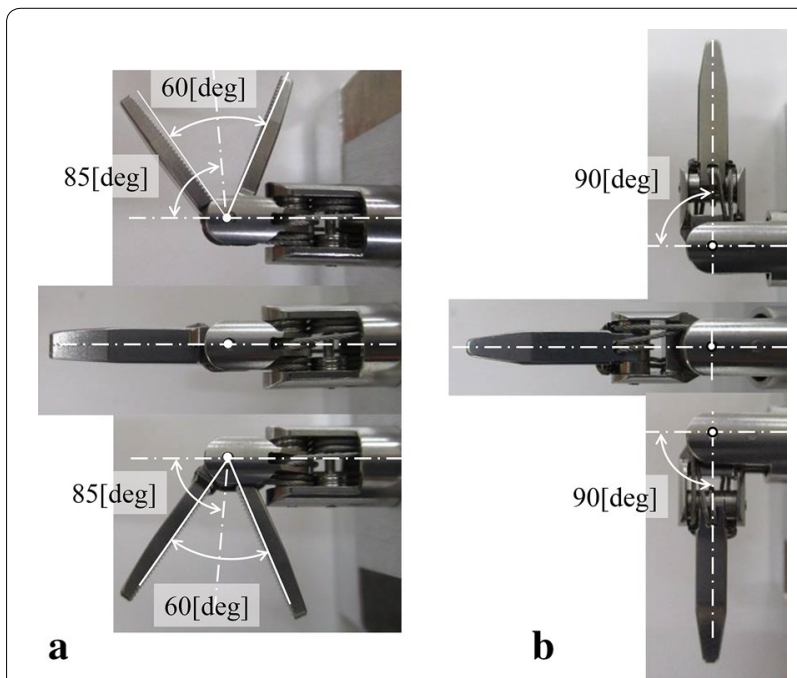

Fig. 7 Range of motion: a yaw and gripper axis range of motion (top view); $\mathbf{b}$ pitch axis range of motion (front view)

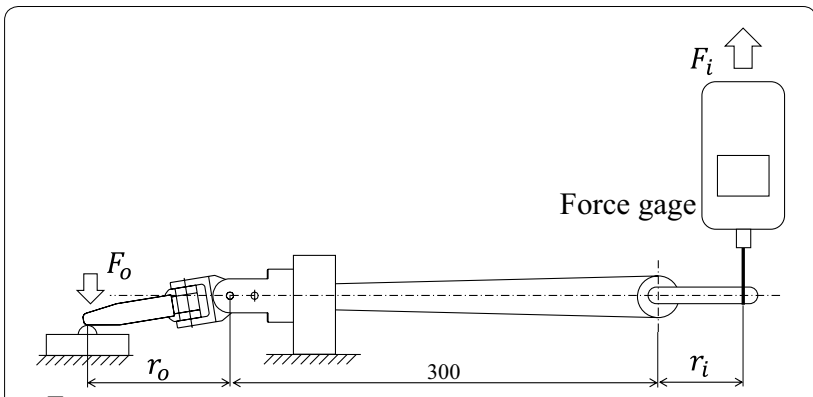

Force sensor

Fig. 8 Measurement drive-mechanism efficiency

Table 5 lists the efficiency of each axis of the wrist mechanism. Various friction forces degrade efficiency: between the shaft and pulley, between the pulley and wires due to the stretching of the wire, and between the wire strands. The mechanism for the first axis is just one pair of wire and pulleys. On the other hand, since the wires of the second and third axis pass through the pitch axis, they rub on the pitch shaft portion, and the efficiencies of the second and third axis are approximately the square of the efficiency of the first axis.

Table 5 Efficiency of the wrist mechanism

\begin{tabular}{ll}
\hline Axis no. & Efficiency (\%) \\
\hline 1st axis & 75.2 \\
2nd axis & 60.7 \\
3rd axis & 55.1 \\
\hline
\end{tabular}

\section{Results of output force and torque}

The motion performance of the wrist mechanism was evaluated with a test bench. Figure 9 shows an overview of the test bench. Servo motors HS-85 BB (Hitec Multiplex Japan, Inc.) were used for the drive motors, and an Arduino Leonardo microcontroller was used. Table 6 lists the specifications of the servo motors. The output (force and torque) for each axis with driving torque applied was measured by the test bench. A force sensor was used in a similar fashion to that seen in Fig. 8. When measuring the second and third axes, the pitch axis was again fixed. Table 7 lists the experimental data and calculated values from a maximum motor torque of $294 \mathrm{mNm}$, along with the measured efficiency listed in Table 5 for output force and torque of each joint. The outputs corresponding to the efficiency of each axis are listed.

As shown in Eq. (4), the grasping force is generated by the 2nd and the 3rd axis torque by cooperative driving mechanism. In case of $\tau_{2}=-\tau_{3}$, the grasping torque $\tau_{g}=\frac{e}{c} \tau_{2}$. Therefore, the grasping force and torque is almost the same as the output force and torque of 2nd and 3rd axis, as shown in Table 7.

Furthermore, tests demonstrated that each motion of the yaw, pitch, and gripper axis can be initiated by a command from the tactile-switch user interface with calculations from the kinematics matrices in Eq. (2). An additional movie file shows this in more detail (see Additional file 1).

These results confirm that the proposed mechanism has the fundamental functions and performance characteristics to be useful as a wrist mechanism of articulated forceps for robot-assisted laparoscopic surgery.

\section{Conclusions}

Considering the need for a compact and maneuverable wrist mechanism for robot-assisted laparoscopic forceps, a wire-driven wrist mechanism with a pitch, yaw, and gripper axis has been proposed. The following design goals were achieved:

1. The offset distance between the pitch and yaw axes was reduced. The offset value, i.e., $5.95 \mathrm{~mm}$, is shorter than maximum outer diameter $7.5 \mathrm{~mm}$ of the wrist mechanism.

2. Singularity configurations of the wrist axes is avoided near the standard working posture by a configuration of pitch and yaw axis.

3. There are few parts, and it is simple and small offset as compared to the previously developed clinical use robotic forceps $[7,10,14,16,17]$.

Furthermore, the effectiveness of the proposed mechanism was verified by tests. The range of pitch motion 


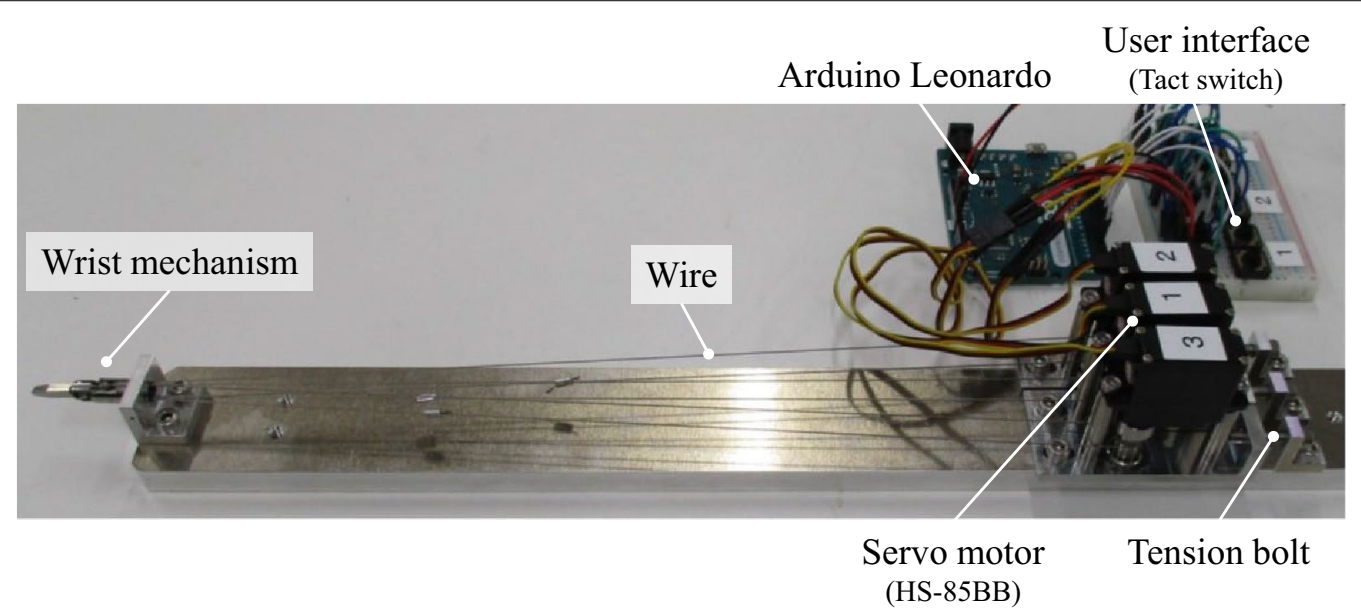

Fig. 9 Test bench

Table 6 Specifications of the servo motor (HS-85BB)

\begin{tabular}{ll}
\hline Item & Specification \\
\hline Operating voltage & $4.8 \mathrm{~V}$ \\
Stall torque & $294 \mathrm{mNm}$ \\
Operating speed at no load & $375^{\circ} / \mathrm{s}(6.54[\mathrm{rad} / \mathrm{s}])$ \\
\hline
\end{tabular}

Table 7 Output force and torque of each joint

\begin{tabular}{lllll}
\hline Axis no. & Experiment data & \multicolumn{2}{l}{$\begin{array}{l}\text { Calculated value } \\
\text { from maximum motor } \\
\text { torque and measured } \\
\text { efficiency }\end{array}$} \\
\cline { 2 - 3 } \cline { 5 - 6 } & Force $(\mathbf{N})$ & Torque $(\mathbf{m N m})$ & Force $(\mathbf{N})$ & Torque $(\mathbf{m N m})$ \\
\hline 1st axis & 6.9 & 138.6 & 6.6 & 132.4 \\
2nd axis & 5.1 & 71.4 & 5.9 & 82.6 \\
3rd axis & 5.0 & 70.0 & 5.4 & 75.0 \\
\hline
\end{tabular}

is $\pm 90^{\circ}$, yaw motion is $\pm 85^{\circ}$ (or $\pm 90^{\circ}$ ), and gripper motion is $60^{\circ}$ (or $50^{\circ}$ ). The transmission efficiencies are from about 55 to $75 \%$. The grasping force and torque are more than $5 \mathrm{~N}$ and $70 \mathrm{mNm}$.

Future works toward the final robot system as follows:

1. Further analysis of wire-pulley drive mechanism and its brush up. For example, connecting method of wire and pulley or wire-pulley friction drive, increase in the joint stiffness, and high durability of the wire around pulley, among others.

2. The implementation of this wrist mechanism to slave manipulators while considering the clinical environment. Especially, sealing mechanism of air tight structure, cleaning method inside forceps, miniatur- ized and modularized attachment mechanism of forceps are very important. Furthermore, the durability, cleaning and sterilization evaluation are the most important items for a reuse forceps in development process of a commercial device.

3. The development of a master-slave manipulator for laparoscopic surgery with the proposed mechanism using a 6-axis vertical articulated robot [26] or a redundant 7-axis vertical articulated robot to optimize motion both inside and outside of patient's abdominal cavity.

\section{Additional files}

Additional file 1. Demonstration motion of each axis by a tactile-switch user interface.

Authors' contributions

The author read and approved the final manuscript.

Acknowledgements

Not applicable.

Competing interests

The author declares that he has no competing interests.

Availability of data and materials Not applicable.

Ethics approval and consent to participate Not applicable.

\section{Funding}

Not applicable.

\section{Publisher's Note}

Springer Nature remains neutral with regard to jurisdictional claims in published maps and institutional affiliations. 
Received: 24 October 2017 Accepted: 27 February 2018

Published online: 05 March 2018

\section{References}

1. Japan Society for Endoscopic Surgery (2016) 13th Nationwide survey of endoscopic surgery in Japan. J Jpn Soc Endosc Surg 21(6):655-810 (in Japanese)

2. Intuitive Surgical, Inc., the da Vinci ${ }^{\circledR}$ Surgical System. http://www.intui tivesurgical.com/. Accessed 16 Oct 2017

3. Cambridge Endoscopic Devices, Inc. Autonomy ${ }^{\mathrm{TM}}$. http://www.cambr idgeendo.com/. Accessed 16 Oct 2017

4. FlexDex Inc. FlexDex, https://flexdex.com/. Accessed 16 Oct 2017

5. Tuebingen Scientific Medical GmbH, Radius Surgical System. http://www. tuebingen-scientific.com/. Accessed 1 Feb 2018

6. Hackethal A, Koppan M, Eskef K, Tinneberg H-R (2012) Handheld articulating laparoscopic instruments driven by robotic technology. First clinical experience in gynecological surgery. Gynecol Surg 9(2):203-206.

7. Wallace DT, Julian CA, Morley TA, Baron DS (2002) Surgical tools for use in minimally invasive telesurgical applications, US Patent 6,394,998, 28 May 2002

8. Thielmann S, Seibold U, Haslinger R, Passig G, Bahls T, Jörg S, Nickl M, Nothhelfer A, Hagn U, Hirzinger G (2010) MICA — a new generation of versatile instruments in robotic surgery. In: Proceedings IEEE/RSJ international conference on intelligent robots and systems, pp 871-878

9. Tadano K, Kawashima K, Kojima K, Tanaka N (2010) Development of a pneumatic surgical manipulator IBIS IV. J Robot Mechatron 22(2):179-188

10. Jinno M (2012) Manipulator, US Patent 8,277,443, 2 Oct 2012

11. Jinno M, Sunaoshi T, Miyagawa T, Hato T, Matsuhira N, Morikawa Y, Ozawa S, Kitajima M (2006) Development of robotic forceps for laparoscopic surgery. J Robot Mechatron 18(3):249-254

12. Jinno M, Sunaoshi T, Omori S (2012) Working mechanical device and manipulator, US Patent 7,942,895, 17 May 2011

13. Nishizawa K, Kishi K (2004) Development of interference-free wire driven joint mechanism for surgical manipulator systems. J Robot Mechatron 16(2):116-121

14. Braun M (2012) Surgical instrument comprising an instrument handle and zero point adjustment, US Patent 8,267,958, 18 Sep 2012
15. Fujii M, Fukushima K, Sugita N, Ishimaru T, Iwanaka T, Mitsuishi M (2011) Design of intuitive user interface for multi-DOF forceps for laparoscopic surgery. In: IEEE international conference on robotics and automation, pp $5743-5748$

16. Lee W, Chamorro A (2008) Surgical instrument, US Patent 7,338,513, 4 Mar 2008

17. Awtar S, Nielsen J, Trutna T, Mansfield A, Abani R, Geiger J, Quigley P (2014) Minimal access tool, US Patent 8,668,702, 11 Mar 2014

18. Haraguchi D, Kanno T, Tadano K, Kawashima K (2015) A pneumaticallydriven surgical manipulator with a flexible distal joint capable of force sensing. IEEE/ASME Trans Mechatron 20(6):2950-2961

19. Yamashita H, Hata N, Hashizume M, Dohi T (2004) Handheld laparoscopic forceps manipulator using multi-slider linkage mechanisms. In: Medical Image Computing and Computer-Assisted Intervention-MICCAI 2004, 7th international conference. Proceedings Part II, pp 121-128

20. Arata J, Mitsuishi M, Warisawa S, Tanaka K, Yoshizawa T, Hashizume M (2005) Development of a dexterous minimally-invasive surgical system with augmented force feedback capability. In: IEEE/RSJ international conference on intelligent robots and systems, pp 3207-3212

21. Reboulet C Durand-Leguay S (1999) Optimal design of redundant parallel mechanism for endoscopic surgery. In: Proceedings IEEE/RSJ international conference on intelligent robots and systems, pp 1432-1437

22. Kobayashi Y, Chiyoda S, Watabe K, Okada M, Nakamura Y (2002) Small occupancy robotic mechanisms for endoscopic surgery. In: Medical Image Computing and Computer-Assisted Intervention-MICCAI 2002, 5th international conference. Proceedings Part I, pp 75-82

23. Hong MB, Jo YH (2014) Design of a novel 4-DOF wrist-type surgical instrument with enhanced rigidity and dexterity. IEEE/ASME Trans Mechatron 19(2):500-511

24. Ishii C, Kobayashi K (2007) Development of a new bending mechanism and its application to robotic forceps manipulator. In: Proceedings IEEE international conference on robotics and automation, pp 238-243

25. Arata J, Saito Y, Fujimoto H (2010) Outer shell type 2 DOF bending manipulator using spring-link mechanism for medical applications. In: Proceedings of IEEE international conference on robotics and automation, pp 1041-1046

26. Jinno M (2014) Master-slave manipulator for laparoscopic surgery using a 6-axis vertical articulated robot. ROBOMECH J 1:23

\section{Submit your manuscript to a SpringerOpen ${ }^{\circ}$ journal and benefit from:}

- Convenient online submission

- Rigorous peer review

- Open access: articles freely available online

- High visibility within the field

- Retaining the copyright to your article

Submit your next manuscript at $\boldsymbol{\nabla}$ springeropen.com 